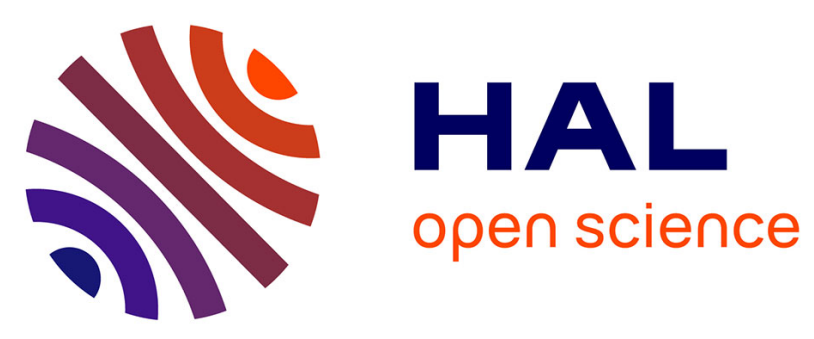

\title{
Conditional Comonotonicity
}

Clotilde Napp, Elyès Jouini

\section{To cite this version:}

Clotilde Napp, Elyès Jouini. Conditional Comonotonicity. Decisions in Economics and Finance, 2005, 27 (2), pp.153-166. halshs-00151516

\section{HAL Id: halshs-00151516 https://shs.hal.science/halshs-00151516}

Submitted on 6 Jun 2007

HAL is a multi-disciplinary open access archive for the deposit and dissemination of scientific research documents, whether they are published or not. The documents may come from teaching and research institutions in France or abroad, or from public or private research centers.
L'archive ouverte pluridisciplinaire HAL, est destinée au dépôt et à la diffusion de documents scientifiques de niveau recherche, publiés ou non, émanant des établissements d'enseignement et de recherche français ou étrangers, des laboratoires publics ou privés. 


\title{
Conditional comonotonicity*
}

\author{
Elyès JOUINI ${ }^{\dagger} \quad$ Clotilde NAPP ${ }^{\ddagger}$
}

July 5, 2004

\begin{abstract}
In this paper we propose a generalization of the comonotonicity notion by introducing and exploring the concept of conditional comonotonicity. We characterize this notion and we show on examples that conditional comonotonicity is the natural extension of the concept of comonotonicity to dynamic settings.
\end{abstract}

\section{Introduction}

The notion of comonotonicity appears quite naturally in decision theory (see Yaari, 1987), finance (see Dybvig, $1988 a$ and $b$ ), insurance and actuarial sciences (see Dhaene et al., $2002 a$ and $b$ ). In this paper, we propose a generalization of this notion by introducing and exploring the concept of conditional comonotonicity. We characterize this notion and we show on examples that conditional comonotonicity is the natural concept when dealing with dynamic settings.

Let us first recall that a subset of $\mathbb{R}^{2}$ is said to be comonotonic if it is completely ordered for the componentwise order ${ }^{1}$ of $\mathbb{R}^{2}$. Now, two random variables defined on the same probability space are said to be comonotonic if the support of the joint distribution is comonotonic.

Characterizations and properties of comonotonic random variables can be found in Denneberg (1994) or Dhaene et al. (2002a). In particular, if two random variables $x$ and $y$ are such that there exists a nondecreasing function $\varphi$ for which $x$ can be written in the form $x=\varphi(y)$ (or if $y$ can be written in the form $y=\varphi(x)$ ), then $x$ and $y$ are comonotonic. In fact, $x$ and $y$ are comonotonic if and only if they are nondecreasing functions of the same third random variable $z$. As underlined by Shaun and Dhaene (1998) comonotonic risks can be then considered as "common monotonic".

\footnotetext{
*The authors want to thank Walter Schachermayer for helpful discussions.

†Corresponding author. CEREMADE, Université de Paris IX Dauphine, Place du Maréchal de Lattre de Tassigny, 75116 Paris and Institut universitaire de France. e-mail : jouini@ceremade.dauphine.fr

${ }^{\ddagger}$ CEREMADE, Université de Paris IX Dauphine, Place du Maréchal de Lattre de Tassigny, 75116 Paris and CREST. e-mail : napp@ceremade.dauphine.fr.

${ }^{1}$ For two vectors $x$ and $y$ of $\mathbb{R}^{2}$, this order is defined by $x \leq y$ if $x_{i} \leq y_{i}, i=1,2$.
} 
This concept of comonotonicity emerges naturally in social choice theory since as proved by Landsberger and Meilijson (1994), all Pareto optimal (for the second order stochastic dominance) risk allocations are comonotonic. In particular, in insurance, most classical risk sharing schemes between insurer and reinsurer or between insured and insurer lead to partial risks that are comonotonic. From the individual point of view, the optimal consumption plan of a utility maximizing agent, under a given budget constraint, is anticomonotonic with the state price density ${ }^{2}$. Dybvig (1988a and $b$ ) elaborates on this property to introduce the concept of efficient strategies and inefficiency costs. More generally, the concept of comonotonicity has revealed to be very useful and has been extensively studied and applied (see e.g. Denneberg, 1994, Dhaene et al., 2002 $a$ and $b$, Landsberger and Meilijson, 1994, Chateauneuf et al., 1996, Carlier and Dana, 2002).

However the comonotonicity concept is not in general adapted to dynamic settings. In particular, we shall see that the comonotonicity of Pareto optimal allocations is no longer satisfied in a general dynamic setting. More precisely we show that when the agents maximize a discounted sum of their expected utility for future consumption with a path-dependent utility function (e.g. a habit formation utility function) and/or a stochastic discount rate the Pareto optimal allocations are no more comonotonic but conditionally comonotonic. Similarly, the optimal consumption plan of a utility maximizing agent, under a given budget constraint, is no more anticomonotonic with the state price density but only, in some sense, conditionally anticomonotonic. Analogously, in insurance, as soon as we consider a dynamic, "realistic enough" setting, we are led to introduce a weaker form of comonotonicity. Indeed, many contracts encountered in insurance like quota share coverage (resp. coverage with maximal limit, resp. stop-loss coverage) in which one agent takes a risk equal to $\alpha x$ (resp. $\min (x, \ell)$, resp. $(x-d)^{+}$) where $x$ represents the total risk and $\alpha$ (resp. $\ell$, resp. $d$ ) is a given proportion (resp. maximal limit, resp. franchise) would lead to comonotonic risks if $\alpha$ (resp. $\ell$, resp. $d$ ) was constant which is not the case in practice. In practice, dynamic risk-sharing contracts permit to adapt dynamically the proportion $\alpha$, the limit $\ell$ or the franchise $d$ depending on the previous claims. Loosely speaking, in these dynamic settings, for the insurance examples as well as for the Pareto optimal allocations or for the utility maximizing agent, the comonotonicity is satisfied at a given date $t$ but only from date $t-1$ point of view. We propose in this paper to introduce, characterize and explore the implications of such a generalization of the usual concept of comonotonicity. In a natural way, we shall call this concept conditional comonotonicity.

The paper is organized as follows. We start in Section 2 by recalling the standard notion of comonotonicity, its definition and its main different characterizations and properties. We then give a formal definition of conditional comonotonicity. We provide different characterizations and properties of conditional comonotonic random variables, which are the "conditional analog" of the well known characterizations and properties of (simply) comonotonic random

\footnotetext{
2 i.e. comonotonic with the opposite of the state price density.
} 
variables. Section 3 deals with examples and applications of the notion of conditional comonotonicity. We show that the concept of conditional comonotonicity appears as the natural extension of the concept of comonotonicity as soon as we deal with dynamic settings. We consider the examples of habit-formation utility functions and utility functions with a stochastic discount rates. Furthermore, we show that the concept of conditional comonotonicity is particularly adapted in order to analyze the impact of subjective beliefs on the risk-premium in a CCAPM-like framework. More precisely, we show that a subjective probability that has a density conditionally anticomonotonic with the total wealth can be interpreted as a pessimistic one and that such a pessimistic subjective belief leads to an increase of the risk premium. These results are consistent with those of Abel (2002) or Cecchetti et al. (2000) obtained in various settings with various definitions of the concept of pessimism. All these definitions have in common that, loosely speaking, they put more weight on bad (resp. good) states of the world. Our conditional comonotonicity property appears then as a well adapted mathematical tool for a formal definition of the concepts of optimism and pessimism in a dynamic framework.

All proofs are in the Appendix.

\section{Conditional comonotonicity}

We consider a probability space $(\Omega, \mathcal{F}, P)$ and a sub sigma-field $\mathcal{G}$ of $\mathcal{F}$. We assume that $\mathcal{F}$ and $\mathcal{G}$ are complete, i.e. that they contain all the zero-probability sets. We shall sometimes assume that the space is endowed with a filtration $\left(\mathcal{F}_{t}\right)_{t=0}^{T}$.

We start by recalling the standard concept of comonotonicity.

Definition 1 A subset $A \subseteq \mathbb{R}^{2}$ is said to be comonotonic if for all $\left(\left(x_{1}, y_{1}\right),\left(x_{2}, y_{2}\right)\right)$ in $A \times A$, we have $\left(x_{1}-y_{1}\right)\left(x_{2}-y_{2}\right) \geq 0$.

So a subset $A \subseteq \mathbb{R}^{2}$ is comonotonic if for any $\left(x_{1}, y_{1}\right)$ and $\left(x_{2}, y_{2}\right)$ in $A$, either $\left(x_{1}, y_{1}\right) \leq\left(x_{2}, y_{2}\right)$ or $\left(x_{1}, y_{1}\right) \geq\left(x_{2}, y_{2}\right)$ holds. This means that for any $\left(x_{1}, y_{1}\right)$ and $\left(x_{2}, y_{2}\right)$ in $A$, if $x_{i}<y_{i}$ for some $i=1,2$, then $\left(x_{1}, y_{1}\right) \leq\left(x_{2}, y_{2}\right)$. Hence a comonotonic set is "simultaneously nondecreasing" in each component.

Before to introduce the definition of comonotonic random variables let us recall that a set $A$ is called a support for a given random variable $Z$ if $P(Z \in A)=$ 1 holds true.

Definition 2 Two random variables $X$ and $Y$ on $(\Omega, \mathcal{F}, P)$ are said to be comonotonic if the law of $(X, Y)$ has a comonotonic support.

Dhaene et al. (2002) obtain the following equivalent characterizations for comonotonicity of two random variables. As usual, for a given random variable $Z$, we let $F_{Z}$ denote its cumulative distribution function. This function is right-continuous and nondecreasing and we define, as usual, its inverse by $F_{Z}^{-1}(p)=\inf \left\{x \in \mathbb{R}: F_{Z}(x) \geq p\right\}$. 
Proposition 1 (Dhaene et al. (2002)) Let $X$ and $Y$ be two random variables on $(\Omega, \mathcal{F}, P)$. The following conditions are equivalent:

1. The law of $(X, Y)$ has a comonotonic support.

2. For all $(x, y) \in \mathbb{R}^{2}$, we have $F_{(X, Y)}(x, y)=\min \left(F_{X}(x), F_{Y}(y)\right)$.

3. We have $(X, Y) \stackrel{d}{=}\left(F_{X}^{-1}(U), F_{Y}^{-1}(U)\right)$ where $U \sim \mathcal{U}_{[0,1]}$, i.e. for any Borel subset $A$ of $\left.\mathbb{R}^{2}, P[(X, Y) \in A]\right)=P\left[\left(F_{X}^{-1}(U), F_{Y}^{-1}(U)\right) \in A\right]$.

4. There exist a random variable $\xi$ and two nondecreasing functions $f$ and $g$ such that $(X, Y) \stackrel{d}{=}(f(\xi), g(\xi))$ i.e., for any Borel subset $A$ of $\mathbb{R}^{2}$, $P[(X, Y) \in A])=P[(f(\xi), g(\xi)) \in A]$.

See also Denneberg (1994) for other characterizations and properties of comonotonic random variables. We propose to generalize this notion of comonotonicity conditionally to $\mathcal{G}$. In the next, we shall denote by $F_{\left.(X, Y)\right|_{\mathcal{G}}}$ the conditional cumulative distribution function defined by $\left.F_{(X, Y) \mid}(x, y)=P[X \leq x, Y \leq y \mid \mathcal{G}]\right)$. We know that there exists a transition kernel $M:(\Omega, \mathcal{F}) \rightarrow\left(\mathbb{R}^{2}, \mathcal{R}^{2}\right)$ such that for all Borel subset $A$ of $\mathbb{R}^{2}, P((X, Y) \in A \mid \mathcal{G})(\omega)=M(\omega, A)$ and $M(\omega,$.$) is$ the conditional law of $(X, Y)$ with respect to $\mathcal{G}$.

Definition 3 Two random variables $X$ and $Y$ on $(\Omega, \mathcal{F}, P)$ are said to be comonotonic conditionally to $\mathcal{G}$ if the conditional law of $(X, Y)$ with respect to $\mathcal{G}$ has a comonotonic support.

Before to elaborate more on this concept let us provide an elementary example of conditionally comonotonic variables that are not comonotonic in the usual sense. Let us take $\Omega=\left\{\omega_{1}, \omega_{2}, \omega_{3}, \omega_{4}\right\}$ and let us consider the random variables $X$ and $Y$ defined by

$\begin{array}{ccc} & X & Y \\ \omega_{1} & 1 & 3 \\ \omega_{2} & 2 & 4 \\ \omega_{3} & 3 & 1 \\ \omega_{4} & 4 & 2\end{array}$.

These two random variables are clearly not comonotonic. However, if we consider the sub-sigma field $\mathcal{G}$ defined by $\mathcal{G}=\left\{\emptyset,\left\{\omega_{1}, \omega_{2}\right\},\left\{\omega_{3}, \omega_{4}\right\}, \Omega\right\}$, then it appears that $X$ and $Y$ are comonotonic conditionally to $\mathcal{G}$.

It is easy to see that if two random variables are comonotonic in the classical sense then they are comonotonic conditionally to any sub-sigma field $\mathcal{G}$. Furthermore, the comonotonicity in the classical sense is equivalent to the comonotonicity conditionally to the trivial sigma-field.

We obtain the "conditional analog" of Proposition 1.

Proposition 2 Let $X$ and $Y$ be two random variables on $(\Omega, \mathcal{F}, P)$. The following conditions are equivalent: 
1. The conditional law of $(X, Y)$ with respect to $\mathcal{G}$ has a comonotonic support.

2. For all $(x, y) \in \mathbb{R}^{2}$, we have $F_{\left.(X, Y)\right|_{\mathcal{G}}}(x, y)=\inf \left(F_{\left.X\right|_{\mathcal{G}}}(x), F_{\left.Y\right|_{\mathcal{G}}}(y)\right)$.

3. We have, for any Borel subset $A$ of $\mathbb{R}^{2}$,

$$
P((X, Y) \in A \mid \mathcal{G})(\omega)=P\left[\left(F_{\left.X\right|_{\mathcal{G}}}^{-1}(U, \omega), F_{\left.Y\right|_{\mathcal{G}}}^{-1}(U, \omega)\right) \in A\right],
$$

where $F_{\left.X\right|_{\mathcal{G}}}^{-1}(., \omega)$ is the inverse function of $F_{\left.X\right|_{\mathcal{G}}}(., \omega)$.

4. There exist a random variable $\xi$ and two functions $f: \Omega \times \mathbb{R} \rightarrow \mathbb{R}$ and $g: \Omega \times \mathbb{R} \rightarrow \mathbb{R}$ such that $f(., x)$ (resp. $g(., x)$ ) is $\mathcal{G}-$ measurable, $f(\omega,$. (resp. $g(\omega,)$.$) is nondecreasing and such that for any Borel subset A$ of $\mathbb{R}^{2}, P((X, Y) \in A \mid \mathcal{G})(\omega)=P[(f(\omega, \xi), g(\omega, \xi)) \in A]$.

The last property means that $(X, Y)$ has the same conditional distribution as $(f(\omega, \xi), g(\omega, \xi))$ for some random variable $\xi$ and some $\mathcal{G}$-measurable with respect to the first variable, nondecreasing with respect to the second variable, functions $f$ and $g$. The next proposition provides an almost sure representation instead of a representation in distribution.

Proposition 3 Let $X$ and $Y$ be two random variables on $(\Omega, \mathcal{F}, P)$. The following conditions are equivalent:

1. The random variables $X$ and $Y$ are comonotonic conditionally to $\mathcal{G}$.

2. There exist a random variable $\xi$ and two functions $f: \Omega \times \mathbb{R} \rightarrow \mathbb{R}$ and $g: \Omega \times \mathbb{R} \rightarrow \mathbb{R}$ such that $f(., x)$ (resp. $g(., x)$ ) is $\mathcal{G}$-measurable, $f(\omega,$. (resp. $g(\omega,)$.$) is nondecreasing and such that (X, Y)=(f(\omega, \xi), g(\omega, \xi))$ $P-a . s$.

3. There exist two functions $f: \Omega \times \mathbb{R} \rightarrow \mathbb{R}$ and $g: \Omega \times \mathbb{R} \rightarrow \mathbb{R}$ such that $f(., x)$ (resp. $g(., x))$ is $\mathcal{G}$-measurable, $f(\omega,).($ resp. $g(\omega,)$.$) is continuous$ and nondecreasing and such that $(X, Y)=(f(\omega, X+Y), g(\omega, X+Y))$ P-a.s.

If we replace in the two last conditions the almost sure equality by an equality for each $\omega$, we would obtain the definition of a non-probabilistic concept of conditional comonotonicity. The non-probabilistic conditional comonotonicity would then correspond to the (probabilistic) conditional comonotonicity with respect to all possible probability measures $P$ on $(\Omega, \mathcal{F})$.

Finally, we shall need in the next section the following generalization of the well known result that if two random variables on $(\Omega, \mathcal{F}, P)$ are comonotonic, then their covariance with respect to any probability measure, absolutely continuous with respect to $P$, is nonnegative (see for instance, Chateauneuf et al., 1996). We denote by $\operatorname{cov}_{\mathcal{G}}^{Q}(X, Y)$ the covariance of $X$ and $Y$ conditionally to $\mathcal{G}$. 
Proposition 4 Let $X$ and $Y$ be two random variables on $(\Omega, \mathcal{F}, P)$. If $X$ and $Y$ are comonotonic conditionally to $\mathcal{G}$, then we have $\operatorname{cov}_{\mathcal{G}}^{Q}(X, Y) \geq 0$ for all probability measure $Q$ absolutely continuous with respect to $P$.

We also introduce the notion of conditionally comonotonic processes.

Definition 4 Two adapted random processes $\left(X_{t}\right)$ and $\left(Z_{t}\right)$ defined on $\left(\Omega, \mathcal{F},\left(\mathcal{F}_{t}\right), P\right)$ are said to be conditionally comonotonic if for $t=0, \cdots, T-1$, the random variables $X_{t+1}$ and $Z_{t+1}$ are comonotonic conditionally to $F_{t}$.

When the uncertainty is described by an event tree, the conditional comonotonicity concept is clear enough: at each date- $t$ node the restriction of $\left(X_{t+1}\right)$ and $\left(Z_{t+1}\right)$ to the immediate successors of the considered node are comonotonic in the classical sense.

With these definitions, it is easy to see that for adapted processes $\left(X_{t}\right),\left(d_{t}\right)$ and $\left(\ell_{t}\right)$, the processes $\left(X_{t}\right),\left(X_{t}-d_{t-1}\right)^{+}, X_{t}-\left(X_{t}-d_{t-1}\right)^{+}, \min \left(X_{t}, \ell_{t-1}\right)$ and $X_{t}-\min \left(X_{t}, \ell_{t-1}\right)$ are conditionally comonotonic.

The following result is then an immediate consequence of Proposition 4

Corollary 1 The product of two conditionally comonotonic martingales (or submartingales) is a submartingale.

In the next section, we show that conditional comonotonicity appears as a particularly well adapted generalization of the usual concept of comonotonicity when dealing with dynamic settings.

\section{Examples and applications}

\subsection{Habit formation utility functions}

Let us consider a utility maximizing agent endowed with the following habitformation utility function

$$
U\left(\left(c_{t}\right)_{t=0, \cdots, T}\right)=E\left[\sum_{t=0}^{T} u\left(c_{t}-x_{t}\right)\right]
$$

where $\left(x_{t}\right)_{t=0, \cdots, T}$ is defined by

$$
x_{0}=0, \quad x_{t+1}=a c_{t}-b x_{t} .
$$

where $a$ and $b$ are given constants with $a \neq b$ and $b \neq 0$ and where $u$ is a concave differentiable utility function. We clearly have

$$
x_{t+1}=a \sum_{k=0}^{t}(-b)^{t-k} c_{k} .
$$

This habit-formation discrete time framework is a slight generalization of the model of Campbell and Cochrane (1999). 
The agent is submitted to a budget constraint given by

$$
E\left[\sum_{t=0}^{T} q_{t} c_{t}\right] \leq \alpha
$$

where $\alpha$ is the agent's initial wealth and where $\left(q_{t}\right)$ is the state-price density process, i.e. $P(\omega) q_{t}(\omega)$ is the price at date 0 (and in terms of date- 0 units of consumption) of one unit of consumption at date $t$ and in the state of the world $\omega$.

The first order necessary conditions for optimality can be written as follows

$$
\begin{array}{r}
u^{\prime}\left(c_{t}-x_{t}\right)-\lambda q_{t}+a \mu_{t}=0 \\
u^{\prime}\left(c_{t}-x_{t}\right)+b \mu_{t}+\mu_{t-1}=0
\end{array}
$$

where the scalar $\lambda$ and the adapted process $\left(\mu_{t}\right)$ are the Lagrange multipliers associated to the optimization program and where $\mu_{-1}=0$. This leads to

$$
\begin{aligned}
\mu_{t} & =\frac{\lambda}{a-b} \sum_{k=0}^{t-1} \frac{q_{t-k}}{(a-b)^{k}}+\frac{1}{(a-b)^{t}} \mu_{0} \\
\mu_{0} & =-\frac{1}{b} u^{\prime}\left(c_{0}\right) \\
u^{\prime}\left(c_{0}\right) & =\frac{\lambda b}{b-a} q_{0}
\end{aligned}
$$

and finally,

$$
c_{t}=\left(u^{\prime}\right)^{-1}\left[\frac{\lambda}{b-a}\left(b q_{t}+a \sum_{k=1}^{t} \frac{q_{t-k}}{(a-b)^{k}}\right)\right]+a \sum_{k=0}^{t-1}(-b)^{t-k} c_{k}
$$

The multiplier $\lambda$ is determined by the budget constraint.

From the concavity of $u$ it is clear then that $\left(c_{t}\right)$ is conditionally comonotonic to $\left(-\frac{\lambda b}{b-a} q_{t}\right)$. From the condition $u^{\prime}\left(c_{0}\right)=\frac{\lambda b}{b-a} q_{0}$ we obtain that $\frac{\lambda b}{b-a}>0$ and $\left(c_{t}\right)$ is then conditionally comonotonic to $\left(-q_{t}\right)$.

A simple example shows that we do not have the (global) comonotonicity between $\left(c_{t}\right)$ and $\left(q_{t}\right)$. Let us assume that $a=0.1, b=-0.9, u(c)=\log c$ and let us chose $\alpha$ in order to have $\lambda=1$. Finally, let us take the price process $q$ as follows

$\begin{array}{cccc}q & t=0 & t=1 & t=2 \\ \omega_{1} & 1 & 2 & 4 \\ \omega_{2} & 1 & 2 & 1.001 \\ \omega_{3} & 1 & 0.5 & 1 \\ \omega_{4} & 1 & 0.5 & 0.25\end{array}$

We obtain then, at $t=2, c_{2}\left(\omega_{1}\right) \simeq 0.47, c_{2}\left(\omega_{2}\right) \simeq 1.83, c_{2}\left(\omega_{3}\right) \simeq 1.73$, $c_{2}\left(\omega_{4}\right) \simeq 13.73$ 
The conditional comonotonicity appears then as more adapted than the classical (global) comonotonicity to this habit-formation framework.

If we assume now that we have $n$ agents $i=1, \cdots, n$ with utility functions defined as previously by $u_{i}, a_{i}$ and $b_{i}$, it is clear that at the equilibrium all the individual allocations will be conditionally comonotonic. An extension of the previous example to a two agents framework permits to show that the equilibrium allocations are not necessarily (globally) comonotonic among the agents. Indeed, let us take the same utility function for both agents $u(c)=\log c$, the same price process as in the previous example, the same parameter $\lambda=1$ for both agents and $a_{1}=0.1, b_{1}=-0.9, a_{2}=0.01, b_{2}=-0.99$. We obtain then the following allocations

\begin{tabular}{ccccccc} 
agents & \multicolumn{3}{c}{$i=1$} & & & $i=2$ \\
$\omega \backslash t$ & $t=0$ & $t=1$ & $t=2$ & $t=0$ & $t=1$ & $t=2$ \\
$\omega_{1}$ & 1,1111 & 0,6993 & 0,4730 & 1,0101 & 0,5177 & 0,2796 \\
$\omega_{2}$ & 1,1111 & 0,6993 & 1,8341 & 1,0101 & 0,5177 & 1,0557 \\
$\omega_{3}$ & 1,1111 & 2,9683 & 1,7302 & 1,0101 & 2,0720 & 1,0564 \\
$\omega_{4}$ & 1,1111 & 2,9683 & 13,7302 & 1,0101 & 2,0720 & 4,3318
\end{tabular}

In fact, agent 2 puts less weight on past consumption in order to evaluate present utility. This weight on past consumption is sufficiently small in order to have as in the classical expected utility framework that $c^{2}$ is comonotonic with $-q$ (or equivalently anticomonotonic with $q$ ) which is not the case for $c^{1}$.

It is easy to prove that the conditional comonotonicity property is satisfied by any Pareto optimal allocation. Furthermore, the equilibrium allocation above is an example of Pareto optimal allocation that is not (globally) comonotonic.

\subsection{Expected utility functions with a stochastic discount factor}

The same properties as in the habit-formation framework can be obtained in a classical expected utility framework if we represent the psychological discount factor by a stochastic and predictable process (instead of a scalar as in the standard framework).

Indeed, let us consider an agent maximizing the following utility function

$$
U\left(\left(c_{t}\right)_{t=0, \cdots, T}\right)=E\left[\sum_{t=0}^{T} \beta_{t} u\left(c_{t}\right)\right]
$$

under the budget constraint

$$
E\left[\sum_{t=0}^{T} q_{t} c_{t}\right] \leq \alpha
$$

where the adapted process $\left(q_{t}\right)$ is the state price density process, where $\alpha$ is the initial wealth and where $\left(\beta_{t}\right)$ is a predictable psychological discount factor. 
The first order conditions leads to

$$
\beta_{t} u^{\prime}\left(c_{t}\right)=\lambda q_{t}, \quad \text { for all } t
$$

where $\lambda$ is the Lagrange multiplier associated with the budget constraint.

Since $\left(\beta_{t}\right)$ is predictable and $u^{\prime}$ is decreasing, the consumption process appears immediately as conditionally anticomonotonic with the price process. If we assume as previously that $u(c)=\log c, \lambda=1$ and that the price process and the discount process are given by

$\begin{array}{cccccccc}q & t=0 & t=1 & t=2 & \beta & t=0 & t=1 & t=2 \\ \omega_{1} & 1 & 2 & 4 & \omega_{1} & 1 & 1 & 1.05 \\ \omega_{2} & 1 & 2 & 1.001 & \omega_{2} & 1 & 1 & 1.05 \\ \omega_{3} & 1 & 0.5 & 1 & \omega_{3} & 1 & 1 & 1 \\ \omega_{4} & 1 & 0.5 & 0.25 & \omega_{4} & 1 & 1 & 1\end{array}$

we obtain

$\begin{array}{cccc}c & t=0 & t=1 & t=2 \\ \omega_{1} & 1 & 0.5 & 0.2625 \\ \omega_{2} & 1 & 0.5 & 1.0490 \\ \omega_{3} & 1 & 2 & 1 \\ \omega_{4} & 1 & 2 & 4\end{array}$

and it appears then that the consumption process is not necessarily (globally) anticomonotonic with the price process.

If we now introduce a second agent with the same characteristics except that he has a constant (equal to 1) discount factor, we obtain the following equilibrium allocation

\begin{tabular}{ccccccc} 
agents & \multicolumn{3}{c}{$i=1$} & \multicolumn{3}{c}{$i=2$} \\
$\omega \backslash t$ & $t=0$ & $t=1$ & $t=2$ & $t=0$ & $t=1$ & $t=2$ \\
$\omega_{1}$ & 1 & 0.5 & 0.2625 & 1 & 0.5 & 0.25 \\
$\omega_{2}$ & 1 & 0.5 & 1.0490 & 1 & 0.5 & 0,990 \\
$\omega_{3}$ & 1 & 2 & 1 & 1 & 2 & 1 \\
$\omega_{4}$ & 1 & 2 & 4 & 1 & 2 & 4
\end{tabular}

and the individual allocations are then obviously conditionally comonotonic but are not (globally) comonotonic.

\subsection{CCAPM and pessimism}

An immediate consequence of Proposition 4 is the following

Corollary 2 For positive random variables $X$ and $Z$ such that $X$ is comonotonic with $Z$ conditionally to $F_{t}$, we have

$$
\operatorname{cov}_{t}^{P}\left[\frac{X Y}{E_{t}^{P}[X Y]}, Z\right] \geq \operatorname{cov}_{t}^{P}\left[\frac{Y}{E_{t}^{P}[Y]}, Z\right]
$$

for any positive random variable $Y$. 
A possible application of this result is related to the Consumption based Capital Asset Pricing Model (Ingersoll, 1987, Huang and Litzenberger, 1988, Duffie, 1996). This formula provides a relationship, valid at the equilibrium, between the excess expected return of a given asset and the covariance between these returns and the marginal utility of the representative agent. More precisely, we have

$$
E_{t}^{P}\left[R_{t+1}\right]-r_{t+1}^{f}=-\operatorname{cov}_{t}^{P}\left[\frac{v^{\prime}\left(t+1, e_{t+1}\right)}{E_{t}^{P}\left[v^{\prime}\left(t+1, e_{t+1}\right)\right]}, R_{t+1}\right]
$$

where $r_{t+1}^{f}$ is the risk-free rate between date $t$ and date $t+1, R_{t+1}$ is the return of the considered asset on the same period, $e_{t+1}$ is the total wealth at date $t+1$ and $v$ is the utility function of the representative agent.

It has been often argued that investors might have a "subjective belief/opinion" reflecting their pessimism/optimism. Consider then a slight extension of the standard model and suppose that the representative agent maximizes his expected utility from future consumption under a subjective probability $Q$, equivalent to $P$. The representative agent maximizes

$$
U\left(\left(c_{t}\right)_{t=0, \cdots, T}\right)=E^{Q}\left[\sum_{t=0}^{T} u\left(c_{t}\right)\right]
$$

under the budget constraint

$$
E\left[\sum_{t=0}^{T} q_{t} c_{t}\right] \leq \alpha .
$$

It is easy to verify that the CCAPM formula becomes then

$$
E_{t}^{P}\left[R_{t+1}\right]-r_{t+1}^{f}=-\operatorname{cov}_{t}^{P}\left[\frac{M_{t+1} v^{\prime}\left(t+1, e_{t+1}\right)}{E_{t}^{P}\left[M_{t+1} v^{\prime}\left(t+1, e_{t+1}\right)\right.}, R_{t+1}\right]
$$

where $\left(M_{t}\right)$ is the density process of $Q$ with respect to $P$

There are different ways to define a pessimistic belief. It seems natural to relate the definition of a pessimistic belief to the fact that it puts more weight (with respect to the true probability) on bad states of the world (bad returns) and less weight on good states of the world (good returns). We propose the following definition.

Definition 5 A probability belief $Q$ equivalent to $P$, with density process $\left(M_{t}\right)$ is said to be pessimistic (resp. optimistic) (with respect to the returns process $\left.\left(R_{t}\right)\right)$ if $\left(M_{t}\right)$ is conditionally anticomonotonic (resp. comonotonic) with $\left(R_{t}\right)$.

Under $Q$ the expected return between date $t$ and date $t+1$, from date $t$ point of view is given by $E_{t}^{Q}\left[R_{t+1}\right]=E_{t}^{P}\left[\frac{M_{t+1}}{M_{t}} R_{t+1}\right]$ and by Proposition 4 , we have $E_{t}^{Q}\left[R_{t+1}\right] \leq E_{t}^{P}\left[\frac{M_{t+1}}{M_{t}}\right] E_{t}^{P}\left[R_{t+1}\right]=E_{t}^{P}\left[R_{t+1}\right]$. The expected return 
under the pessimistic probability is then lower than under the objective initial probability which is a natural property for a pessimistic belief.

The following corollary is then an immediate consequence of Corollary 2.

Corollary 3 The expected excess return predicted by the CCAPM in a pessimistic beliefs setting is higher than in the standard setting.

In an optimistic beliefs setting we would have the opposite result. The impact of the introduction of a subjective belief on the market price of risk is then very clear: it leads to an increase (resp. decrease) of the market price of risk of a given asset (with respect to the standard setting) if the subjective probability is pessimistic (resp. optimistic), where pessimistic means, in particular, that the instantaneous rate of return of the considered asset (under this probability) is lower than under the objective initial probability. Our results are consistent with those of Abel (2002), Cechetti et al. (2000), Chauveau and Nalpas (1998) or Hansen et al. (1999) which introduce distorted beliefs associated to cautious/pessimistic individual behavior.

\section{Appendix}

\section{Proof of Proposition 2}

1 . $\Rightarrow 2$. If the support of $M(\omega,$.$) is comonotonic, we have$

$$
\begin{aligned}
F_{\left.(X, Y)\right|_{\mathcal{G}}}(x, y) & =P[X \leq x, Y \leq y \mid \mathcal{G}]) \\
& =M(\cdot,]-\infty ; x] \times]-\infty ; y]) \\
& =\inf \{M(\cdot,]-\infty ; x] \times]-\infty ;+\infty]), M(\cdot,]-\infty ;+\infty] \times]-\infty ; y])\} .
\end{aligned}
$$

2. $\Rightarrow 3$. It suffices to prove the equality for all Borelian set of the form $A=]-\infty ; x] \times]-\infty ; y]$. We have

$$
\begin{aligned}
& \left.\left.\left.\left.P\left[\left(F_{\left.X\right|_{\mathcal{G}}}^{-1}(U, \omega), F_{\left.Y\right|_{\mathcal{G}}}^{-1}(U, \omega)\right) \in\right]-\infty ; x\right] \times\right]-\infty ; y\right]\right] \\
& =P\left[U \leq F_{\left.X\right|_{\mathcal{G}}}(x, \omega), U \leq F_{\left.Y\right|_{\mathcal{G}}}(y, \omega)\right] \\
& =P\left[U \leq \inf \left(F_{\left.X\right|_{\mathcal{G}}}(x, \omega), F_{\left.Y\right|_{\mathcal{G}}}(y, \omega)\right)\right] \\
& =\inf \left(F_{\left.X\right|_{\mathcal{G}}}(x, \omega), F_{\left.Y\right|_{\mathcal{G}}}(y, \omega)\right) .
\end{aligned}
$$

3. $\Rightarrow$ 4. We take $\xi=U, f(\omega, s)=F_{\left.X\right|_{\mathcal{G}}}^{-1}(s, \omega)$ and $g(\omega, t)=F_{\left.Y\right|_{\mathcal{G}}}^{-1}(t, \omega)$.

4. $\Rightarrow 1$. The set $\left\{\left(f\left(\omega, \xi\left(\omega^{\prime}\right)\right), g\left(\omega, \xi\left(\omega^{\prime}\right)\right)\right) ; \omega^{\prime} \in \Omega\right\} \subseteq \mathbb{R}^{2}$ is comonotonic.

\section{Proof of Proposition 3}

1 . $\Rightarrow 2$. From Proposition 2 , there exist a random variable $\xi$ and two functions $f: \Omega \times \mathbb{R} \rightarrow \mathbb{R}$ and $g: \Omega \times \mathbb{R} \rightarrow \mathbb{R}$ such that $f(., x)$ (resp. $g(., x)$ ) is $\mathcal{G}$-measurable, $f(\omega,$.$) (resp. g(\omega,$.$) ) is nondecreasing and such that for$ any Borel subset $A$ of $\mathbb{R}^{2}, P((X, Y) \in A \mid \mathcal{G})(\omega)=P[(f(\omega, \xi), g(\omega, \xi)) \in A]$ or equivalently $P\left(\left(f^{-1}(\omega, X), g^{-1}(\omega, Y)\right) \in A \mid \mathcal{G}\right)=P[(\xi, \xi) \in A]$. Consequently, 
we have $P\left(f^{-1}(\omega, X)=g^{-1}(\omega, Y) \mid \mathcal{G}\right)=1, P$-a.s. and then $P\left(f^{-1}(\omega, X)=\right.$ $\left.g^{-1}(\omega, Y)\right)=1$. Let us define the random variable $\tilde{\xi}$ by $\tilde{\xi}(\omega)=f^{-1}(\omega, X(\omega))$, we have $(X, Y)=(f(\omega, \tilde{\xi}), g(\omega, \tilde{\xi})), P$-a.s.

2. $\Rightarrow 3$. For a given $\omega,(a, b) \rightarrow a+b$ is an homeomorphism between $\{(f(\omega, x), g(\omega, x)): x \in \mathbb{R}\}$ and $\{(f(\omega, x)+g(\omega, x)): x \in \mathbb{R}\}$. Indeed, if $f(\omega, x)+$ $g(\omega, x)=f(\omega, y)+g(\omega, y)$ with $x>y$ we have $f(\omega, x) \geq f(\omega, y)$ and $g(\omega, x) \geq$ $g(\omega, y)$ and then $f(\omega, x)=f(\omega, y)$ and $g(\omega, x)=g(\omega, y)$. Let us denote $\varphi_{\omega}$ the converse application. We have $\varphi_{\omega}: f(\omega, x)+g(\omega, x) \rightarrow(f(\omega, x), g(\omega, x))$ and $\varphi_{\omega}$ can be written on the form $(\tilde{f}(\omega,),. \tilde{g}(\omega,)$.$) . We have then (X, Y)=$ $(\tilde{f}(\omega, X+Y), \tilde{g}(\omega, X+Y)), P-$ a.s.

$3 . \Rightarrow 1$. Immediate.

\section{Proof of Proposition 4}

We have

$$
\begin{aligned}
\operatorname{cov}_{t}^{Q}(X, Y)(\omega) & =E_{t}[X Y](\omega)-E_{t}[X](\omega) E_{t}[Y](\omega) \\
& =\int x_{1} y_{1} M\left(\omega, d\left(x_{1}, y_{1}\right)\right) \otimes M\left(\omega, d\left(x_{2}, y_{2}\right)\right) \\
& -\frac{1}{2} \int x_{1} y_{2}^{\prime} M\left(\omega, d\left(x_{1}, y_{1}\right)\right) \otimes M\left(\omega, d\left(x_{2}, y_{2}\right)\right) \\
& -\frac{1}{2} \int x_{2} y_{1} M\left(\omega, d\left(x_{1}, y_{1}\right)\right) \otimes M\left(\omega, d\left(x_{2}, y_{2}\right)\right) \\
& =\int\left(x_{1}-x_{2}\right)\left(y_{1}-y_{2}\right) M\left(\omega, d\left(x_{1}, y_{1}\right)\right) \otimes M\left(\omega, d\left(x_{2}, y_{2}\right)\right)
\end{aligned}
$$

which is nonnegative since the conditional law of $(X, Y)$ with respect to $\mathcal{G}$ have a comonotonic support.

\section{Proof of Corollary 1}

If $\left(X_{t}\right)$ and $\left(Y_{t}\right)$ are conditionally comonotonic martingales (or submartingales), we have

$$
\begin{aligned}
E_{t-1}\left[X_{t} Y_{t}\right] & =E_{t-1}\left(X_{t}\right) E_{t-1}\left(Y_{t}\right)+\operatorname{cov}_{t-1}\left(X_{t} Y_{t}\right) \\
& \geq X_{t-1} Y_{t-1}
\end{aligned}
$$

\section{Proof of Corollary 2}

This inequality is equivalent to

$$
E_{t}^{P}\left[\frac{X Y Z}{E_{t}^{P}[X Y]}\right] \geq E_{t}^{P}\left[\frac{Y Z}{E_{t}^{P}[Y]}\right]
$$

or to

$$
E_{t}^{Q}\left[\frac{X Z}{E_{t}^{Q}[X]}\right] \geq E_{t}^{Q}[Z]
$$

and finally to $\operatorname{cov}_{t}^{Q}(X, Z) \geq 0$, where $Q$ is the probability defined by $\frac{d Q}{d P}=$ $\frac{Y}{E_{t}^{P}(Y)}$. Proposition 4 concludes. 


\section{References}

[1] Abel, A., 2002. An exploration of the effects of pessimism and doubt on asset returns. Journal of Economic Dynamics and Control, 26, 1075-1092.

[2] Campbell, J., Cochrane, J., 1999. By force of habit : a consumption based explanation of aggregate stock market behavior, Journal of Political Economy, 102, 205-251

[3] Carlier, G., and R.-A. Dana, 2002, "Core of convex distortions of a probability on a non atomic space" to appear in Journal of Economic Theory.

[4] Cecchetti, S.G., Lam, P., and N.C. Mark, 2000. Asset pricing with distorted beliefs: are equity returns too good to be true? American Economic Review, 90, 787-805.

[5] Chateauneuf, A., R. Kast and A. Lapied (1996). Choquet pricing for financial markets with frictions. Mathematical Finance 6, 323-330.

[6] Chauveau, T, Nalpas, N., 1998. L'altération prudente des probabilités comme solution à l'énigme de la prime de risque. Working Paper, Caisse des Dépôts et Consignations, Service des Etudes Economiques et Financières, Paris.

[7] Dhaene, J., Denuit, M., Goovaerts, M.J., Kaas, R., Vyncke, D., 2002 The concept of comonotonicity in actuarial science and finance : theory. Insurance, Mathematics and Economics, 31, 3-33

[8] Dhaene, J., Denuit, M., Goovaerts, M.J., Kaas, R., Vyncke, D., 2002 The concept of comonotonicity in actuarial science and finance : applications. Insurance, Mathematics and Economics, 31, 133-161

[9] Denneberg, D., 1994 Non additive measure and integral, Kluwer Academic Publishers.

[10] Duffie, D., 1996. Dynamic Asset Pricing Theory. Princeton University Press, Princeton.

[11] Dybvig, P., 1988a Distributional analysis of portfolio choice, Journal of Business, 61, 360-393

[12] Dybvig, P., 1988b, Inefficient Dynamic Portfolio Strategies or How to Throw Away a Million Dollars in the Stock Market, The Review of Financial Studies, 1, 67-88.

[13] Hansen, L.-P., Sargent, T. and T. Tallarini, 1999. Robust Permanent Income and Pricing. Review of Economic Studies, 66, 873-907.

[14] Huang, C.-F., Litzenberger, R., 1988. Foundations of Financial Economics, Prentice Hall, Englewood Cliffs, New-Jersey. 
[15] Ingersoll, J., 1987. Theory of Financial Decision Making, Rowman and Littlefield, Totowa, New-Jersey.

[16] Landsberger, M., Meilijson, I., 1994, "Co-monotone allocations, BickelLehmann dispersion and the Arrow-Pratt measure of risk aversion", Annals of Operations Research, 52, 323-343.

[17] Shaun, W., and Dhaene, J., 1998, "Comonotonicity, correlation order and premium principles", Insurance: Mathematics and Economics, 22, 3, 235242

[18] Yaari, M.E., 1987 The dual theory of choice under risk. Econometrica, 55, 95-115. 